\title{
L-Arginine Supplementation Causes Additional Effects on Exercise- Induced Angiogenesis and VEGF Expression in the Heart and Hind-Leg Muscles of Middle-Aged Rats
}

\author{
Junichi SUZUKI \\ Laboratory of Sports Physiology, Research and Education Center for Winter Sports, Hokkaido University of Education, Ainosato 5-3, \\ Kita-ku, Sapporo, Hokkaido, 002-8502 Japan
}

\begin{abstract}
The effects of dietary L-arginine supplementation on exercise-induced angiogenesis and VEGF expression were examined in male middle-aged (12 months old) Wistar rats. Exercise training lasted for six weeks at $20 \mathrm{~m} / \mathrm{min}$ on a $0 \%$ gradient for 10-60 min/day. Rats in the L-arginine-treated groups drank water containing $2.5 \% \mathrm{~L}$-arginine. According to histochemical identification of the capillary profile, in the soleus muscle the capillary-to-fiber (C:F) ratio showed a significantly greater value in the L-arginine-treated training group than in both the sedentary control and training groups. Training with L-arginine significantly increased the C:F ratio in the subendocardium of the left ventricle, whereas training alone did not. In the plantaris muscle, training with or without L-arginine significantly increased the cap-
\end{abstract}

illary density, but it did not affect the C:F ratio. A Western blot analysis showed that training with L-arginine significantly increased VEGF protein expression by 2.9 -fold in the soleus muscle and by 1.7 -fold in the left ventricle, but the increase with training alone was insignificant. The tissue endothelial nitric oxide synthase protein levels were significantly increased in both the soleus (by 1.3-fold) and the left ventricle (by 1.4-fold) only after training with L-arginine supplementation. In the plantaris muscle, these protein levels did not change after either training or L-arginine treatment. The present results suggest that in middle-aged rats, L-arginine administration caused additional effects on exercise-induced angiogenesis by presumably promoting VEGF expression in the hind-leg muscle as well as in the left ventricle.

Key words: L-arginine, aging, angiogenesis, endurance training, vascular endothelial growth factor.

M any studies have demonstrated that endurance exercise training induces a marked increase in capillarity in skeletal [1] and cardiac muscles [2]. The effects of aging on exercise-induced angiogenesis have been reported in humans, as well as in rats. In elderly humans, Denis et al. [3] first reported that endurance training did not improve capillary supply in skeletal muscle. However, recent findings indicated that skeletal muscle in the elderly could adapt to exercise training by enhancing capillary supply $[4,5]$. In aged rats, a recent study showed that endurance running at maximum tolerated speeds caused marked capillary angiogenesis, and that an increase in vascular endothelial growth factor (VEGF) gene expression in response to acute exercise was not diminished by aging [6]. However, endurance training failed to cause capillary angiogenesis, classified as no increase in the capillary-to-fiber (C:F) ratio, in middle-aged [7] and old rats [8] when the intensity of training was moderate.

$V E G F$ is quite likely an important regulator of exercise-induced angiogenesis [9]. Hypoxia enhanced $V E G F$ gene expression [10]. Recently several other factors, including nitric oxide (NO), have been shown to regulate it. The inhibition of NO synthase (NOS) abolished capillary angiogenesis induced by exercise training in skeletal muscle [11]. Myocardial capillary density and $V E G F$ mRNA expression in endothelial NOS-deficient (eNOS ${ }^{-/}$) mice were markedly less than those in wild-type mice [12]. Although the mRNA level and protein expression of $V E G F$ in ischemic skeletal muscle showed no apparent difference between eNOS $-1-$ and control wild-type mice, ischemia-induced angiogenesis was severely inhibited in eNOS $^{-/-}$mice [13]. Thus endogenous NO plays a critical role in capillary angiogenesis in skeletal muscles, as well as in the heart.

Treatment by chronic L-arginine, a substrate for NOS, enhanced exercise-induced endothelial NO synthesis and aerobic capacity [14]. In a previous study, daily L-arginine supplementation caused additional effects on exercise-induced angiogenesis by promoting VEGF expression in the left ventricles of young rats [15]. Therefore it appears pos-

Received on Oct 26, 2005; accepted on Jan 25, 2006; released online on Feb 4, 2006; DOI: 10.2170/physiolsci.RP000505 Correspondence should be addressed to: Junichi Suzuki, Research and Education Center for Winter Sports Hokkaido University of Education, Ainosato 5-3, Kita-ku, Sapporo, Hokkaido, 002-8502 Japan. Tel: +81-11-778-0614, Fax: +81-11-778-0638, E-mail: suzuki@sap.hokkyodai.ac.jp 
sible that in middle-aged rats, training with L-arginine supplementation causes marked angiogenesis, even though training at moderate intensity itself fails to do so.

In the present study, experiments were designed to examine the combined effects of exercise training and Larginine supplementation on capillary angiogenesis and VEGF protein expression in the left ventricle and hind-leg muscles of middle-aged rats. The results demonstrate that L-arginine administration caused additional effects on exercise-induced angiogenesis by promoting VEGF expression in the hind-leg muscles, as well as in the left ventricle.

\section{METHODS}

This study was approved by the Animal Care and Use Committee of Hokkaido University of Education and performed in accordance with the "Guiding Principles for the Care and Use of Animals in the Field of Physiological Sciences" of the Physiological Society of Japan.

Animals, experimental conditions, and muscle samples. Twenty-four (10 months old) male Wistar rats were purchased from Clea Japan Inc. (Tokyo, Japan). After the rats were fed for two months to allow adaptation to the new environment, they were randomly divided into the following three groups according to their treatment and exercise conditions: nontreated sedentary ( $\mathrm{Cnt}, n=8)$, nontreated training (TR, $n=8$ ), and L-arginine-treated training (Ar$\mathrm{TR}, n=8)$. The L-arginine administration and exercise training started at 12 months of age and lasted for 6 weeks. During the last week of the adaptation period, all rats were subjected to treadmill running for two min per day for three days. They were housed under controlled temperature conditions $\left(24 \pm 1^{\circ} \mathrm{C}\right)$ and a relative humidity of approximately 50\%. Lighting (07:00-19:00) was controlled automatically. All rats were given commercial laboratory chow (CE-2, Clea Japan Inc.) ad libitum. Rats in the nontreated groups drank tap water, whereas rats in the L-arginine-treated groups drank water containing $2.5 \%(\mathrm{w} / \mathrm{v}) \mathrm{L}-$ arginine (A5006, Sigma-Aldrich, St. Louis, USA). The average L-arginine intake, estimated from water intake, was $2.24 \mathrm{~g} / \mathrm{kg} /$ day. Since the rats were housed two to three per cage, the water intake per individual rat was estimated from the water intake per cage.

The training groups were subjected to running on a rodent treadmill (KN-73, Natume Co., Tokyo, Japan). The rats ran $10 \mathrm{~min}$ per day at $20 \mathrm{~m} / \mathrm{min}$ with a $0 \%$ gradient on the first day of training. Then the duration was increased by three min per day. The speed and gradient were maintained throughout the remaining training period. At the end of the 4th week, the rats were running continuously for $1 \mathrm{~h}$ per day. This final duration was maintained throughout the remaining training period. The training session was carried out five days per week.
The animals were not exercised for at least $48 \mathrm{~h}$ prior to killing. Under light anesthesia with ether, the rats were anesthetized with $\alpha$-chloralose $(0.06 \mathrm{~g} / \mathrm{kg}$ I.P.) and urethane $(0.7 \mathrm{~g} / \mathrm{kg}$ I.P. $)$. A toe-pinch response was used to validate adequate anesthesia. Then the left soleus (SOL) and plantaris (PL) muscles and left ventricle (LV) were excised and weighed. The muscles were fixed at the length measured when the knee joint was maximally extended, and the tibiotarsal joint was fixed at 90 degrees. The tissues were placed in embedding medium, an Optimal Cutting Temperature (O.C.T.) compound (Sakura Finetechnical, Tokyo, Japan), and rapidly frozen in isopentane cooled to its melting point $\left(-160^{\circ} \mathrm{C}\right)$ with liquid nitrogen. For biochemical analyses, the remaining muscles, i.e., the right side, were excised and frozen in liquid nitrogen. The apex half of LV was used for biochemical analyses, and the other half was used for histochemical analyses. All LV samples were treated in a similar fashion. The tissue samples were stored at $-80^{\circ} \mathrm{C}$ until analyses.

Histological analyses. Tissue cross sections, $10 \mu \mathrm{m}$ thick, were obtained by use of a cryotome (CM-1500; Leica Japan, Tokyo, Japan) at $-20^{\circ} \mathrm{C}$. To determine capillary profiles, the sections were double stained for alkaline phosphatase (AP) and dipeptidyl peptidase IV (DPP IV) in the capillary endothelium. Although the original staining protocol was described by Lojda [16], a slightly modified protocol was used, as previously described [17]. The images of incubated sections were digitized with a digital microscope camera (PDMC le, Polaroid, Cambridge, USA), attached to a light microscope (BX-50, Olympus, Tokyo, Japan), and stored on computer disk. Nonoverlapping microscopic fields were selected at random from each muscle sample. During the measurements, the observer was blind in regard to the source (groups) of each slide. Morphological analyses were obtained from the SOL, the mixed fiber portion of the PL, and the LV subendocardium. Total cross-sectional areas used for each morphological measurement were up to $0.69 \mathrm{~mm}^{2}$ per tissue region.

Western blot analysis. Tissue homogenates were obtained from approximately $50 \mathrm{mg}$ of frozen tissue homogenized for three interrupted $15 \mathrm{~s}$ bursts wim phosphate buffer, $\mathrm{pH} 7.2 ; 0.1 \%$ Triton $\mathrm{X}-100 ; 1 \mathrm{mM}$ dithiothreitol; $5 \%[\mathrm{v} / \mathrm{v}]$ protease inhibitor cocktail [P2714, Sigma]). After centrifugation at $1,500 \times g$ for $15 \mathrm{~min}$ at $0^{\circ} \mathrm{C}$, the supernatant was used for protein analysis. A portion $(20 \mu \mathrm{g})$ of protein was fractionated by SDS/PAGE on 7.5 or $12 \%(\mathrm{w} /$ v) polyacrylamide gels, electrically transferred to a polyvinylidene fluoride membrane and blocked with $1 \%(\mathrm{w} / \mathrm{v})$ bovine serum albumin for $1 \mathrm{~h}$. The blots were exposed to the specific primary antibodies (Santa Cruz Biotech, Santa Cruz, CA, USA) against VEGF $(1: 1,000)$ or eNOS $(1: 1,000)$ diluted in blocking solution. After the blots were incubated with an AP-conjugated secondary antibody (Santa Cruz, 1:5,000) diluted in blocking solution, the 
Table 1. Body and organ mass values.

\begin{tabular}{lcrc}
\hline & Cnt $(n=8)$ & TR $(n=8)$ & ArTR $(n=8)$ \\
\hline Initial body mass $(\mathrm{g})$ & $570.0 \pm 18.6$ & $579.6 \pm 13.0$ & $586.9 \pm 16.1$ \\
Final body mass $(\mathrm{g})$ & $612.4 \pm 21.2$ & $598.3 \pm 15.2$ & $575.4 \pm 18.0$ \\
Body mass gain $(\mathrm{g})$ & $42.4 \pm 4.3$ & $18.6 \pm 9.8^{*}$ & $-11.5 \pm 6.2^{*} \#$ \\
Body mass gain (\%) & $7.4 \pm 0.7$ & $3.3 \pm 1.7^{*}$ & $-2.0 \pm 1.0^{*} \#$ \\
Organ mass (mg) & & \\
$\quad$ Whole heart & $1,407.2 \pm 40.4$ & $1,394.0 \pm 49.8$ & $1,399.3 \pm 45.9$ \\
$\quad$ Left ventricle & $973.9 \pm 28.3$ & $968.9 \pm 46.8$ & $970.5 \pm 33.5$ \\
$\quad$ Soleus & $212.9 \pm 9.2$ & $219.0 \pm 2.3$ & $218.8 \pm 6.8$ \\
$\quad$ Plantaris & $686.3 \pm 29.6$ & $666.0 \pm 16.8$ & $679.1 \pm 31.0$ \\
Organ mass-to-body mass ratio (mg/g) & & & \\
$\quad$ Whole heart & $2.30 \pm 0.04$ & $2.33 \pm 0.05$ & $2.43 \pm 0.03^{*}$ \\
$\quad$ Left ventricle & $1.60 \pm 0.04$ & $1.62 \pm 0.05$ & $1.69 \pm 0.04$ \\
$\quad$ Soleus & $0.35 \pm 0.01$ & $0.37 \pm 0.01$ & $0.38 \pm 0.01^{*}$ \\
$\quad$ Plantaris & $1.12 \pm 0.03$ & $1.12 \pm 0.02$ & $1.18 \pm 0.04$ \\
\hline Values & &
\end{tabular}

Values are means \pm SE. ${ }^{*}$ and \#, significantly different from Cnt and TR, respectively, at $p<0.05$.

rquired proteins were detected with a 5-bromo-4-chloro3 -indolyl phosphate/nitro blue tetrazolium reaction. The membranes were scanned (GT-8200UF, Seiko Epson Co., Tokyo, Japan), and densities of bands were quantified, using Image J public domain software (written by W. Rasband, National Institutes of Health, USA). Negative controls without primary or secondary antibodies were run to validate the results.

Biochemical analyses. Metabolic enzyme activities were measured by the use of muscle homogenate aliquots. The activity of $\beta$-hydroxyacyl-CoA-dehydrogenase (HAD) was assayed according to the method of Bass et al. [18]. The activity of citrate synthase (CS) was determined according to the methods of Srere [19]. All measurements were carried out at $25^{\circ} \mathrm{C}$ with a spectrophotometer (U2001, Hitachi Co., Tokyo, Japan).

Statistical analyses. All values are expressed as means \pm SE.The distribution of all parameters was tested by the use of Kolmogrov-Smirnov test to determine whether it was compatible with a normal distribution. The differences among the three groups were analyzed with one-way ANOVA and Fisher's PLSD post hoc tests. The differences were considered statistically significant at $P<0.05$.

\section{RESULTS}

Mean body mass at the end of treatment was not significantly different among the three groups (Table 1). Body mass gain during the experimental period was significantly less in the TR and ArTR groups than in the Cnt group $(p<0.05)$. Moreover, training with L-arginine reduced body mass less than training alone $\operatorname{did}(p<0.05)$. Training did not affect the tissue mass or its mass-to-body-mass ratio in all organs examined in the present study, whereas training with L-arginine significantly increased the tissue mass-to-body mass ratio in the whole heart and soleus muscle (SOL; $p<0.05$ ). Dietary L-arginine supplementation caused additional effects on body mass reduction and
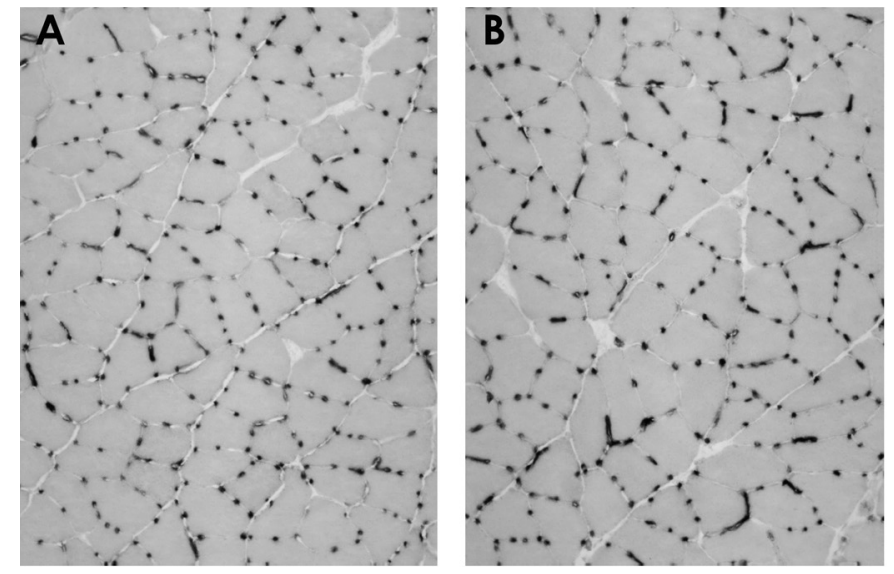

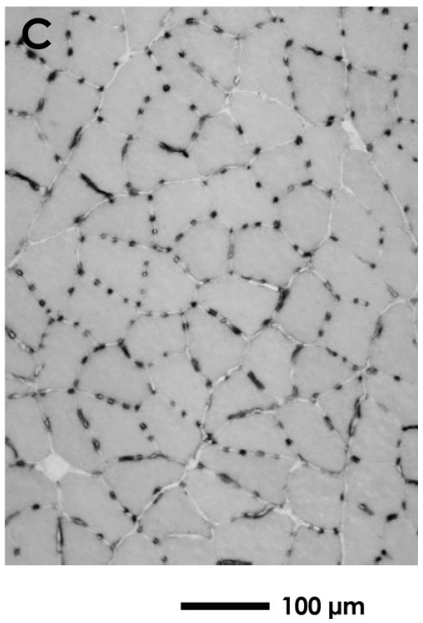

Fig. 1. Micrographic images of the soleus muscle stained with alkaline phosphatase and dipeptidylpeptidase IV. (A) Nontreated sedentary group. (B) Trained group. (C) L-arginine-treated trained group. All images are at the same magnification. 

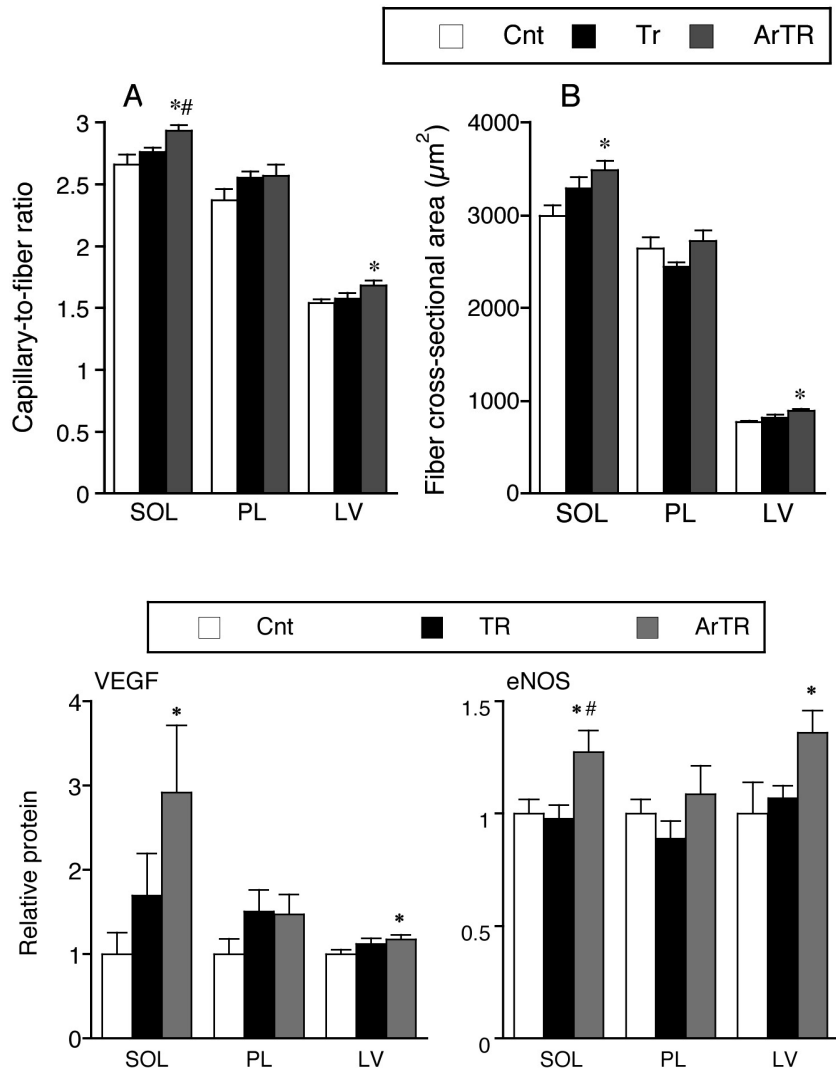

Fig. 3. Western blot analysis demonstrating effects of exercise training or L-arginine supplementation on VEGF and eNOS protein expressions. All values are represented as means \pm SE. * and \#, significantly different from the control (Cnt) and trained (TR) groups, respectively, at $p<0.05$. ArTR, L-arginine-treated trained groups.

tissue hypertrophy induced by exercise training.

An enzyme histochemical identification of capillary profiles was performed to investigate the effects of L-arginine supplementation on exercise-induced microvascular remodeling. Figure 1 shows representative micrographic images of sections that demonstrate capillary and myofiber profiles from the SOL. In the SOL, the capillary-to-fiber $(\mathrm{C}: \mathrm{F})$ ratio showed a significantly higher value in the ArTR than in both the Cnt and TR groups $(p<0.05$; Fig. $2 \mathrm{~A})$. In the LV, training with L-arginine significantly increased the $\mathrm{C}: \mathrm{F}$ ratio $(p<0.05)$, whereas training alone did not. Thus L-arginine treatment caused an additional effect on exercise-induced capillary angiogenesis in hindleg muscle, as well as in the heart. Because of a significantly greater fiber cross-sectional area in the ArTR group ( $p<0.05$; Fig. 2B), a concomitant, but not significant, decrease in capillary density (CD) was also observed in both the SOL and LV (Fig. 2C). In the PL, training with or without L-arginine significantly increased the $\mathrm{CD}(p<$ 0.05 ), whereas it did not affect the C:F ratio. Therefore in hind-leg muscles, the additional effects of L-arginine on exercise-induced angiogenesis were observed in highly oxidative muscle.
Fig. 2. Capillary-to-fiber ratio (A), fiber cross sectional area (B), and capillary density (C) in the soleus (SOL), mixed-fiber portion of plantaris (PL), and subendocardium of left ventricle (LV). All values are represented as means \pm SE. * and \#, significantly different from the control (Cnt) and trained (TR) groups, respectively, at $p<$ 0.05 . ArTR, L-arginine-treated trained groups.

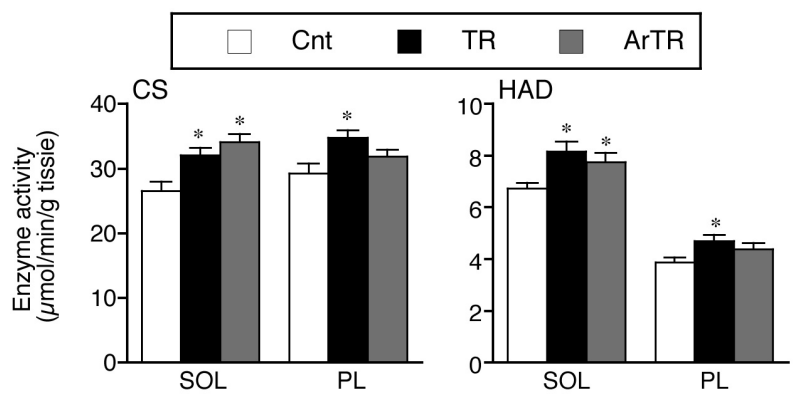

Fig. 4. Enzyme activities in the soleus and plantaris muscles. All values are represented as means $\pm \mathrm{SE}$. ${ }^{*}$, significantly different from the control (Cnt) group at $p<0.05$; CS, citrate synthase; HAD, $\beta$-hydroxyacyl-CoA dehydrogenase.

A Western blot analysis was performed to investigate VEGF and eNOS protein expressions (Fig. 3). In the SOL, although VEGF protein levels were higher in both the TR (1.7-fold) and ArTR (2.9-fold) groups than in the control group, a significant difference was observed only in the ArTR group $(p<0.05)$. Training with L-arginine administration significantly increased VEGF protein expression in the LV $(p<0.05)$, but training alone did not increase it significantly. Tissue levels of eNOS showed a significantly higher value in the ArTR (1.3-fold) than in both the Cnt and TR groups in the SOL $(p<0.05)$. In the LV, a marked increase in eNOS expression (1.4-fold) was observed after training with L-arginine $(p<0.05)$. In the PL, these protein levels did not change after either training or L-arginine treatment. Training with L-arginine supplementation thus enhanced expressions of both VEGF and eNOS proteins in highly oxidative muscles.

Metabolic enzyme activities were determined to investigate the effects of L-arginine administration on muscle metabolism (Fig. 4). Training with or without L-arginine significantly increased CS and HAD activities in the SOL $(p<0.05)$. Although in the PL the activities showed the same changes as observed in the SOL, a significant increase was observed only after training alone $(p<0.05)$. Therefore L-arginine supplementation did not affect enzyme activities. 


\section{DISCUSSION}

Effects of exercise training. In the present study, exercise training alone did not cause capillary angiogenesis in the skeletal muscles of middle-aged rats. This finding coincides with that of a previous study, showing that in the soleus muscle, endurance training caused marked capillary angiogenesis in young rats, but not in middle-aged ones [7]. The intensity of exercise used in the present study can be estimated at approximately $65 \%$ of maximum oxygen consumption $\left(\mathrm{VO}_{2} \max \right)$ in 2-month-old Wistar rats [20]. When the age-related decrease in $V \mathrm{O}_{2 \text { max }}$ is taken into account [21], the intensity would be approximately $75 \%$ of $V \mathrm{O}_{2}$ max for middle-aged rats. In old Fisher 344 rats $(25$ months old), endurance running at $75 \%$ of maximal exercise capacity over 10 weeks failed to increase capillarity in hind-leg muscles, but it did increase capillarity in young (6 months old) rats [8]. A recent investigation showed that, in old Fisher 344 rats (24 months old), endurance running at maximum tolerated speeds for 8 weeks caused marked capillary angiogenesis in hind-leg muscles [6]. Moreover, in their study aging did not impair the increase in VEGF gene expression in hind-leg muscles after acute exercise. Thus it seems possible that in rats, endurance training at maximal intensity causes capillary angiogenesis, whereas training at moderate intensity does not.

Endurance exercise training used in the present study failed to cause angiogenesis in the left ventricle of middleaged rats (Fig. 2). Exercise training beginning in rats younger than 5 weeks induced a marked increase in capillarity, whereas that beginning in those older than 12 weeks did not cause capillary growth $[2,15]$. It seems very likely that moderate-intensity endurance training started in middle-aged or older rats does not improve capillarity in the skeletal muscle and heart.

Effects of I-arginine supplementation. The present study showed that in middle-aged rats, training itself did not cause angiogenesis, whereas training with L-arginine supplementation caused marked exercise-induced angiogenesis in the hind-leg muscles and heart, possibly by promoting VEGF expression.

In the present study, the effect of L-arginine supplementation in the rat without exercise training was not investigated. A previous study indicated that L-arginine administration alone did not affect capillary angiogenesis or VEGF expression in the heart and hind-leg muscles in the young rat [15].

VEGF plays a critical role in exercise-induced angiogenesis [9]. Recent studies have shown that endogenous NO may modulate VEGF-induced angiogenesis [22]. Cardiac capillary density and VEGF mRNA expression were markedly lower in NOS $^{-/-}$than in wild-type mice [12]. The inhibition of NOS diminished the exercise-induced increase in mRNAs for VEGF [23] and VEGFR-1 [24] and in protein levels of VEGF and VEGFR-2 [25] in rat skeletal muscles. Moreover, NOS inhibition failed to cause capillary angiogenesis induced by chronic electrical stimulation in rat skeletal muscle $[11,25]$. Thus the enhanced eNOS expression observed in the present study may have caused capillary angiogenesis by promoting the VEGF system in both the skeletal and cardiac muscles.

In the present study, L-arginine supplementation facilitated capillary angiogenesis and VEGF expression in the SOL and LV, but not in the PL. The soleus muscle is composed of type I (approximately $85 \%$ ) and IIa (15\%) fibers, whereas the mixed fiber portion of plantaris muscle consists of type I (17\%), IIa (27\%), and IIb (56\%) fibers [26]. The intensity and type of exercise used in the present study may have activated predominantly the type I and type IIa fibers. Thus L-arginine may promote exercise-induced angiogenesis in muscles primarily responsible for daily exercise.

In a previous study, L-arginine supplementation with or without training failed to increase eNOS protein in young rats [15]. The protein levels of eNOS in abdominal aorta were markedly less in middle-aged rats (23 months old) than in young ones (4 months) [27]. In skeletal and cardiac muscles, tissue eNOS protein levels were also less in middle-aged than in young rats (Suzuki, unpublished observation). VEGF can promote eNOS expression through VEGFR-2 and PKC [22]. In the present study, both VEGF and eNOS proteins were upregulated in the SOL and LV, but not in the PL (Fig. 3). Thus L-arginine supplementation may help to restore eNOS protein levels in aged rats, possibly via VEGF upregulation.

Mechanical factors during exercise, such as shear stress and tissue stretching, may stimulate the expression of VEGF [28, 29]. Chronic prazosin treatment caused a 4fold increase in shear stress and increased VEGF protein expression in rat skeletal muscle [29]. A recent study showed that shear stress activated the VEGFR-2 pathway, independent of VEGF [30]. Handgrip exercise training with L-arginine administration caused an additional effect on acetylcholine (Ach)-induced hyperemia both in healthy subjects and in patients with heart failure, whereas it improved exercise hyperemia in the patients, but not in healthy subjects [31]. Thus it is unlikely that L-arginine supplementation increased exercise hyperemia and facilitated flow-induced VEGF expression during exercise in the present study.

A single acute bout of exercise increased the expression of VEGF mRNA [32], whereas chronic exercise training diminished its expression after acute exercise in human skeletal muscle [33]. In rat skeletal muscles, the expression of VEGF protein at capillary sites was significantly increased on the 6th and 10th days of the training [17], whereas it still showed a high value after five weeks of training, but was not significantly different from a sedentary control [26]. In the present study, the VEGF level was still significantly higher after six weeks of training 
with L-arginine (Fig. 3). Thus the combined effects of exercise and L-arginine administration can sustain VEGF expression for longer periods. This finding coincides with that of a previous study using young (3-month-old) rats [15].

The infusion of L-arginine enhanced plasma growth hormone [34] and glucagon levels [35]. Since these hormones are known to facilitate lipolysis, L-arginine supplementation may possibly affect fat metabolism in skeletal muscle. Although L-arginine supplementation facilitated body mass reduction during training periods (Table 1), it did not affect the enzyme activity of $\beta$-oxidation in hindleg muscles (Fig. 4). Further investigations are needed to clarify this issue.

In summary, the present study has shown that L-arginine supplementation caused additional effects on exerciseinduced capillary angiogenesis in the hind-leg muscle and left ventricle. The combined effect of exercise and L-arginine induced a marked increase in VEGF and eNOS protein expressions in both tissues. The present results suggest that in middle-aged rats, exercise training with Larginine administration causes capillary angiogenesis in the hind-leg muscles, as well as in the heart, by facilitating VEGF expression.

This work was supported in part by grants-in-aid from the Hokkaido University of Education.

\section{REFERENCES}

1. Hermansen L, Wachtlova M. Capillary density of skeletal muscle in well-trained and untrained men. J Appl Physiol 1971;30:860-3.

2. Jacobs TB, Bell RD, McClements JD. Exercise, age and the development of the myocardial vasculature. Growth. 1984;48:148-57.

3. Denis C, Chatard JC, Dormois D, Linossier MT, Geyssant, Lacour JR. Effects of endurance training on capillary supply of human skeletal muscle on two age groups (20 and 60 years). J Physiol (Paris). 1986;81:379-83.

4. Hepple RT, Mackinnon SL, Goodman JM, Thomas SG, Plyley MJ. Resistance and aerobic training in older men: effects on $\mathrm{VO}_{2 \text { peak }}$ and the capillary supply to skeletal muscle. J Appl Physiol. 1997;82:1305-10.

5. Charifi N, Kadi F, Feasson L, Costes F, Geyssant A, Denis C. Enhancement of microvessel tortuosity in the vastus lateralis muscle of old men in response to endurance training. J Physiol. 2003;554:559-569.

6. Rossiter HB, Howlett RA, Holcombe HH, Entin PL, Wagner HE, Wagner PD. Age is no barrier to muscle structural, biochemical and angiogenic adaptations to training up to 24 months in female rats. J Physiol. 2005;565:993-1005.

7. Suzuki J, Gao M, Batra S, Koyama T. Effects of treadmill training on the arteriolar and venular portions of capillary in soleus muscle of young and middle-aged rats. Acta Physiol Scand. 1997;159:113-21.

8. Mitchell ML, Byrnes WC, Mazzeo RS. A comparison of skeletal muscle morphology with training between young and old Fischer 344 rats. Mech Ageing Dev. 1990;58:21-35.

9. Jensen L, Bangsbo J, Hellsten Y. Effect of high intensity training on capillarization and presence of angiogenic factors in human skeletal muscle. J Physiol. 2004;557:571-82.

10. Goldberg MA, Schneider TJ. Similarities between the oxygen-sensing mechanisms regulating the expression of vascular endothelial growth factor and erythropoietin. J Biol Chem. 1994;269:4355-9.

11. Hudlicka $O$, Brown MD, Silgram H. Inhibition of capillary growth in chronically stimulated rat muscles by N(G)-nitro-L-arginine, nitric oxide synthase inhibitor. Microvasc Res. 2000;5.9:45-51

12. Zhao X, Lu Z, Feng Q. Deficiency in endothelial nitric oxide synthase impairs myocardial angiognesis. Am J Physiol Heart Circ Physiol. 2002;283:H2371-8.

13. Murohara T, Asahara T, Silver M, Bauters C, Masuda H, Kalka C, Kearney M, Chen D, Symes JF, Fishman MC, Huang PL, Isner JM. Nitric oxide synthase modulates angiogenesis in response to tissue ischemia. J Clin Invest. 1998; 101:2567-78.

14. Maxwell AJ, Ho HV, Le CQ, Lin PS, Bernstein D, Cooke JP. L-arginine enhances aerobic exercise capacity in association with augmented nitric oxide production. J Appl Physiol. 2001;90:933-8.

15. Suzuki J. Microvascular angioadaptation after endurance training with $L$-arginine supplementation in rat heart and hindleg muscles. Exp Physiol. 2005;90:763-71.

16. Lojda Z. Studies on dipeptidyl (amino) peptidase IV (glycyl proline norphthylamidase). II. Blood vessels. Histochemistry. 1979;59:153-66.

17. Suzuki J. Time-course changes in VEGF expression and capillarity in the early stage of exercise training with $\mathrm{Co}^{2+}$ treatment in rat skeletal muscle. Acta Physiol Scand. 2004;181:225-32.

18. Bass A, Brdiczka D, Eyer P, Hofer S, Pette D. Metabolic differentiation of distinct muscle types at the level of enzymatic organization. Eur J Biochem. 1969:10:198-206.

19. Srere PA. Citrate synthase. Methods Enzymol. 1969;13:3-5.

20. Brooks GA, White TP. Determination of metabolic and heart rate responses of rats to treadmill exercise. J Appl Physiol. 1978;45:1009-15.

21. Mazzeo RS, Brooks GA, Horvath SM. Effects of age on metabolic responses to endurance training in rats. J Appl Physiol. 1984;57:1369-74.

22. Kimura $H$, Esumi $H$. Reciprocal regulation between nitric oxide and vascular endothelial growth factor in angiogenesis. Acta Biochem Pol 2003;50:49-59.

23. Gavin TP, Spector DA, Wagner H, Breen E, Wagner PD. Nitric oxide synthase inhibition attenuates the skeletal muscle VEGF mRNA response to exercise. J Appl Physiol. 2000;88:1192-8.

24. Gavin TP, Wagner PD. Attenuation of the exercise-induced increase in skeletal muscle Flt-1 mRNA by nitric oxide synthase inhibition. Acta Physiol Scand. 2002;175:201-9.

25. Milkiewicz M, Hudlicka O, Brown MD, Silgram H. Nitric oxide, VEGF and VEGFR-2: interactions in activity-induced angiogenesis in rat skeletal muscle. Am J Physiol Heart Circ Physiol. 2005;289:H336-43.

26. Suzuki J. Microvascular remodelling after endurance training with $\mathrm{CO}^{2+}$ treatment in the rat diaphragm and hind-leg muscles. Jpn J Physiol. 2002;52:409-19.

27. Tanabe T, Maeda S, Miyauchi T, lemitsu M, Takanashi M, Irukayama-Tomobe Y, Yokota T, Ohmori H, Matsuda M. Exercise training improves ageing-induced decrease in eNOS expression of the aorta. Acta Physiol Scand. 2003;178:3-10.

28. Ziada AM, Huclicka O, Tyler KR, Wright AJ. The effect of long-term vasodilation on capillary growth and performance in rabbit heart and skeletal muscle. Cardiovasc Res. 1984;18:724-32.

29. Milkiewicz M, Brown MD, Egginton S, Hudlicka O. Association between shear stress, angiogenesis, and VEGF in skeletal muscles in vivo. Microcirculation. 2001;8:229-41.

30. Wang Y, Chang J, Li YC, Li YS, Shyy JY, Chien S. Shear stress and VEGF activate IKK via the Flk-1/Cbl/Akt signaling pathway. Am J Physiol Heart Circ Physiol. 2004;286:H685-92.

31. Kubota T, Imaizumi T, Oyama J, Ando S, Takeshita A. L-arginine increases exercise-induced vasodilation of the forearm in patients with heart failure. Jpn Circ J. 1997;61:471-80.

32. Breen EC, Johnson EC, Wagner $H$, Tseng HM, Sung LA, Wagner PD. Angiogenic growth factor $m R N A$ responses in muscle to a single bout of exercise. J Appl Physiol. 81996;1:355-61.

33. Richardson RS, Wagner H, Mudaliar SRD, Saucedo E, Henry R, Wagner PD. Exercise adaptation attenuates VEGF gene expression in human skeletal muscle. Am J Physiol Heart Circ Physiol. 2000;279:H772-8.

34. Korbonits M, Trainer PJ, Fanciulli G, Oliva O, Pala A, Dettori A, Besser M, Delitala G, Grossman AB. L-arginine is unlikely to exert neuroendocrine effects in human via the generation of nitric oxide. Eur J Endocrinol. 1996;135:543-7.

35. MacAllister RJ, Calver AL, Collier J, Edwards CM, Herreros B, Nussey SS, Vallance $P$. Vascular and hormonal responses to arginine: provision of substrate for nitric oxide or non-specific effect? Clin Sci (Lond). 1995;89:183-90. 